\title{
Role of Endovenous Laser Ablation versus Radiofrequency Ablation for Primary Varicose Veins \\ ${ }^{1}$ Hesham Adel Alaa El-Diin, ${ }^{1}$ Hesham Mohamed Omraan, ${ }^{2}$ Amr Nabil Kamel, ${ }^{1}$ Mohamed Ahmed Abd El-Moniem Hagag \\ ${ }^{1}$ Department of General Surgery, Faculty of medicine, Ain Shams University \\ ${ }^{2}$ Department of Vascular Surgery, Faculty of medicine, Ain Shams University
}

*Corresponding author: Mohamed Ahmed Abd El-Moniem Hagag, Mobile: 01126560324, E-mail:

hagagmohamed369@gmail.com

\begin{abstract}
Background: varicose veins, a common problem with effects on quality of life, account for a significant cost burden on the health care system. They are enlarged, tortuous, subcutaneous veins that commonly occur in the legs. Varicose veins are caused by faulty valves and decreased elasticity in the vein walls, which allow blood to backflow and pool. This is known as venous reflux. The affected veins enlarge and appear as green, dark blue or purple protrusions just below the skin's surface.
\end{abstract}

Objective: in this study we aimed to compare short term outcomes and postoperative short term complications between EVLA and RFA.

Patients and Methods: this is an observational prospective study. It was carried out in General Surgery department and Vascular Surgery unit at Ain Shams Hospitals and in Kahraba hospital for Research and Treatment.

Results: there was no significant difference between radiofrequency powered segmental ablation and endovenous laser ablation according to demographic data.

Conclusion: there was no significant difference between radiofrequency powered segmental ablation and endovenous laser ablation according to demographic data.

Keywords: varicose veins, endovenous laser therapy, radiofrequency

\section{INTRODUCTION}

Varicose veins, a common problem with effects on quality of life, account for a significant cost burden on the health care system (1). They are enlarged, tortuous, subcutaneous veins that commonly occur in the legs ${ }^{(2)}$.

Varicose veins are caused by faulty valves and decreased elasticity in the vein walls, which allow blood to backflow and pool. This is known as venous reflux. The affected veins enlarge and appear as green, dark blue or purple protrusions just below the skin's surface (3).

The severity of symptoms associated with varicose veins varies and may include pain, heaviness 'pruritis ، ulceration, skin discoloration and edema.
Severe symptoms include thrombophlebitis, bleeding and venous dermatitis, which often require intervention ${ }^{(4)}$.

A variety of therapies are available for treating varicose veins, including conservative therapies, surgical interventions and nonsurgical intervention. Conservative therapies are commonly recommended in asymptomatic patients or those with mild to moderate symptoms. Surgical interventions generally become necessary when symptoms of varicose veins significantly impinge on the patient's quality of life ${ }^{(5)}$.

Junction ligation with or without vein stripping is generally appropriate when the GSV and SSV have reflux or incompetence is demonstrated on duplex scanning. This intervention is generally performed as an inpatient procedure under general anesthetic. Junction ligation involves 
tying off the vessel at the SFJ or SPJ. Ligation alone usually leads to high rates of varicose vein recurrence; therefore, patients often require after-care treatment, such as sclerotherapy. In most cases, ligation is accompanied by GSV stripping and is generally regarded as the treatment of choice for varicose veins ${ }^{(6)}$.

Two endovenous modalities include radiofrequency ablation (RFA) and endovenous laser therapy (ELT). Both treatments involve inserting a heatgenerating laser fiber or catheter into the incompetent saphenous vein, positioned just below the SFJ or SPJ. Heat is generated through laser (ELT) or radiofrequency (RFA) energy, and as the fiber or catheter is slowly removed down the length of the vein, endothelial and venous wall damage occurs, causing contraction of the vein wall and ultimately destruction of the vessel ${ }^{(7)}$.

\section{AIM OF THE WORK}

In this study we aim to compare short term outcomes and postoperative short term complications between EVLA and RFA.

\section{Patients and Methods}

This is an observational prospective study was carried out in General Surgery department and Vascular Surgery unit at Ain Shams Hospitals and in Kahraba hospital for Research and Treatment. Sample size was calculated using PASS ${ }^{\circledR}$ version 11 program, setting the type-1 error $(œ)$ at 0.05 with a width 0.1 , and power at $80 \%$. Result from previous studies showed that the average success rate for RFA 96.2\% compared to $96.7 \%$ among EVLA patients. Based on this the needed sample is 12 cases per each group (24 total). The study was approved by the Ethics Board of Ain Shams University.

Inclusion Criteria: Males and females patients. Patients age (20-50) and fit for anesthesia. Varicose veins affecting the GSV system. Varicose veins confirmed on duplex ultrasound imaging.
Patients suitable for any of the treatment options.

Exclusion Criteria: Patients with secondary varicose veins due to: Deep vein thrombosis. Abdominal mass. Pregnancy.

\section{Preoperative assessment:}

After Informed written consent, patients were subjected to the following: Clinical evaluation and Duplex assessment.

Clinical evaluation was carried out for all patients according to the following scheme: Detailed history (disfigurement, pain, bleeding, deep venous thrombosis, anticoagulant therapy), Detailed general examinations, Lower limb examination to detect: Distribution of veins affected, Incompetent perforators, and Shape (spider, serpentine or saccular).

Duplex was done as a routine to all patients to detect: Patency of the deep system, Sapheno-femoral or Saphenopopliteal reflux, Presence and number of perforators, Diameter of GSV, and Exclusion of deep venous incompetence.

\section{Statistical analysis}

Recorded data were analyzed using the statistical package for social sciences, version 20.0 (SPSS Inc., Chicago, Illinois, USA). Quantitative data were expressed as mean \pm standard deviation (SD). Qualitative data were expressed as frequency and percentage.

The following tests were done: Independent-samples t-test of significance was used when comparing between two means. One-way analysis of variance (ANOVA) was used when comparing between more than two means. Chisquare $\left(\mathrm{x}^{2}\right)$ test of significance was used in order to compare proportions between two qualitative parameters. The confidence interval was set to $95 \%$ and the margin of error accepted was set to $5 \%$. So, the pvalue was considered significant as the following: Probability (P-value): P-value $\leq 0.05$ was considered significant. $\mathrm{P}$-value $\leq 0.001$ was considered as highly 
significant. P-value $>0.05$ was considered insignificant.

\section{RESULTS}

Table (1): Comparison between groups according to radiofrequency powered segmental ablation and endovenous laser ablation according to demographic data.

\begin{tabular}{|c|c|c|c|c|}
\hline $\begin{array}{l}\text { Demograph } \\
\text { ic Data }\end{array}$ & $\begin{array}{l}\text { RPSA } \\
(\mathrm{N}=12)\end{array}$ & $\begin{array}{l}\text { EVLA } \\
(\mathbf{N}=12)\end{array}$ & $\begin{array}{l}\mathbf{t} / \mathbf{x} 2 \\
\#\end{array}$ & $\begin{array}{l}\text { p- } \\
\text { valu } \\
\text { e }\end{array}$ \\
\hline \multicolumn{5}{|l|}{ Age (years) } \\
\hline Range & $20-60$ & $20-60$ & \multirow{2}{*}{$\begin{array}{l}0.3 \\
48\end{array}$} & \multirow{2}{*}{$\begin{array}{l}0.20 \\
7\end{array}$} \\
\hline Mean \pm SD & $\begin{array}{l}41.48 \pm 6 . \\
22\end{array}$ & $\begin{array}{l}43.05 \pm 6 . \\
46\end{array}$ & & \\
\hline \multicolumn{5}{|l|}{ Sex } \\
\hline Male & $3(25 \%)$ & $\begin{array}{l}4 \\
(33.3 \%) \\
\end{array}$ & \multirow{2}{*}{$\begin{array}{l}1.1 \\
17 \#\end{array}$} & \multirow{2}{*}{$\begin{array}{l}0.66 \\
5\end{array}$} \\
\hline Female & $9(75 \%)$ & $\begin{array}{l}8 \\
(66.7 \%)\end{array}$ & & \\
\hline \multicolumn{5}{|l|}{$\begin{array}{l}\text { BMI } \\
{\left[w t /(h t)^{\wedge} 2\right]}\end{array}$} \\
\hline Range & $18-39$ & $19-43$ & \multirow{2}{*}{$\begin{array}{l}0.4 \\
60\end{array}$} & \multirow{2}{*}{$\begin{array}{l}0.27 \\
4\end{array}$} \\
\hline Mean \pm SD & $\begin{array}{l}26.25 \pm 3 . \\
94\end{array}$ & $\begin{array}{l}27.83 \pm 4 \\
17\end{array}$ & & \\
\hline
\end{tabular}

t-Independent Sample t-test; $x^{2}$ : Chisquare test; $p$-value $>0.05 \mathrm{NS}$

\section{Table (1) shows no statistically significant difference between radiofrequency powered segmental ablation and endovenous laser ablation according to demographic data.}

Table (2): Comparison between groups according to radiofrequency powered segmental ablation and endovenous laser ablation according to CEAP class.

\begin{tabular}{|c|c|c|c|c|}
\hline $\begin{array}{c}\text { CEAP } \\
\text { class }\end{array}$ & $\begin{array}{c}\text { RPSA } \\
(\mathbf{N}=\mathbf{1 2})\end{array}$ & $\begin{array}{c}\text { EVLA } \\
(\mathbf{N}=\mathbf{1 2})\end{array}$ & \multirow{2}{*}{$\mathbf{x}$} & $\begin{array}{c}\text { p- } \\
\text { value }\end{array}$ \\
\cline { 1 - 3 } $\mathrm{C} 1$ & $0(0 \%)$ & $0(0 \%)$ & & \\
\cline { 1 - 3 } $\mathrm{C} 2$ & $3(25 \%)$ & $2(16.7 \%)$ & & \\
\cline { 1 - 3 } $\mathrm{C} 3$ & $8(66.7 \%)$ & $8(66.7 \%)$ & \multirow{2}{*}{1.4} & \multirow{2}{*}{0.844} \\
\cline { 1 - 3 } $\mathrm{C} 4$ & $1(8.3 \%)$ & $2(16.7 \%)$ & & \\
\cline { 1 - 3 } $\mathrm{C} 5$ & $0(0 \%)$ & $0(0 \%)$ & & \\
\cline { 1 - 3 } $\mathrm{C} 6$ & $0(0 \%)$ & $0(0 \%)$ & & \\
\hline
\end{tabular}

$\mathrm{x}^{2}$ : Chi-square test; $\mathrm{p}$-value $>0.05 \mathrm{NS}$
Table (2) shows no statistically significant difference between radiofrequency powered segmental ablation and endovenous laser ablation according to CEAP class.

Table (3): Comparison between groups according to radiofrequency powered segmental ablation and endovenous laser ablation according to length of treated vein (cm).

\begin{tabular}{|l|c|c|c|c|}
\hline $\begin{array}{c}\text { Length of } \\
\text { treated vein, } \\
\mathbf{c m}\end{array}$ & $\begin{array}{c}\text { RPSA } \\
(\mathbf{N}=\mathbf{1 2})\end{array}$ & $\begin{array}{c}\text { EVLA } \\
(\mathbf{N}=\mathbf{1 2})\end{array}$ & $\begin{array}{c}\text { t- } \\
\text { tes } \\
\mathbf{t}\end{array}$ & $\begin{array}{c}\mathbf{p}- \\
\mathbf{v a l} \\
\mathbf{u e}\end{array}$ \\
\hline Range & $16-62$ & $16-58$ & \multirow{2}{*}{1.0} & 0.65 \\
Mean $\pm \mathrm{SD}$ & $40.95 \pm 6$ & $38.85 \pm 5$ & 92 & 0 \\
\hline
\end{tabular}

t-Independent Sample t-test; $p$-value $>0.05$ NS

Table (3) shows no statistically significant difference between radiofrequency powered segmental ablation and endovenous laser ablation according to length of treated vein $(\mathrm{cm})$.

Table (4): Comparison between groups according to radiofrequency powered segmental ablation and endovenous laser ablation according to GSV with incompetent terminal valve.

\begin{tabular}{|c|c|c|c|c|}
\hline & $\begin{array}{c}\text { RPSA } \\
(\mathbf{N}=12)\end{array}$ & $\begin{array}{c}\text { EVLA } \\
(\mathrm{N}=12)\end{array}$ & $\mathbf{x 2}$ & $\begin{array}{c}\text { p- } \\
\text { valu } \\
\text { e }\end{array}$ \\
\hline $\begin{array}{l}\text { GSV with } \\
\text { incompetent } \\
\text { terminal } \\
\text { valve }\end{array}$ & $\begin{array}{c}12 \\
(100 \%)\end{array}$ & $\begin{array}{c}12 \\
(100 \%)\end{array}$ & $\begin{array}{l}0.0 \\
00\end{array}$ & $\begin{array}{c}1.00 \\
0\end{array}$ \\
\hline
\end{tabular}

$\mathrm{x}^{2}$ : Chi-square test; $\mathrm{p}$-value $>0.05 \mathrm{NS}$

Table (4) shows no statistically significant difference between radiofrequency powered segmental ablation and endovenous laser ablation according to GSV with incompetent terminal valve.

Table (5): Comparison between groups according to radiofrequency powered segmental ablation and endovenous laser ablation according to preoperative and postoperative Venous Clinical Severity Score (VCSS). 


\begin{tabular}{|c|c|c|c|c|}
\hline $\begin{array}{c}\text { Venous } \\
\text { Clinical } \\
\text { Severity } \\
\text { Score } \\
\end{array}$ & $\begin{array}{c}\text { RPSA } \\
(\mathbf{N}=12)\end{array}$ & $\begin{array}{l}\text { EVLA } \\
(\mathrm{N}=12)\end{array}$ & t-test & $\begin{array}{c}\text { p- } \\
\text { value }\end{array}$ \\
\hline \multicolumn{5}{|l|}{ Preoperative } \\
\hline Mean \pm SD & $3.89 \pm 0.37$ & $4.01 \pm 0.44$ & \multirow{2}{*}{1.349} & \multirow{2}{*}{0.803} \\
\hline Range & $3.43-4.06$ & $3.66-4.42$ & & \\
\hline \multicolumn{5}{|l|}{$\begin{array}{l}\text { After 1 } \\
\text { month }\end{array}$} \\
\hline Mean \pm SD & $2.05 \pm 0.23$ & $2.04 \pm 0.22$ & \multirow{2}{*}{0.260} & \multirow{2}{*}{0.155} \\
\hline Range & $1.68-2.27$ & $1.62-2.30$ & & \\
\hline \multicolumn{5}{|l|}{$\begin{array}{l}\text { After 3 } \\
\text { months }\end{array}$} \\
\hline Mean \pm SD & $2.19 \pm 0.24$ & $2.09 \pm 0.23$ & \multirow{2}{*}{1.129} & \multirow{2}{*}{0.672} \\
\hline Range & $1.81-2.40$ & $1.71-2.31$ & & \\
\hline
\end{tabular}

t-Independent Sample t-test; $p$-value $>0.05$ NS

Table (5) shows no statistically significant difference between radiofrequency powered segmental ablation and endovenous laser ablation according to venous clinical severity score.

Table (6): Comparison between groups according to radiofrequency powered segmental ablation and endovenous laser ablation according to pain score.

\begin{tabular}{|c|c|c|c|c|}
\hline $\begin{array}{l}\text { Pain } \\
\text { Score }\end{array}$ & $\begin{array}{c}\text { RPSA } \\
(\mathrm{N}=12)\end{array}$ & $\begin{array}{c}\text { EVLA } \\
(\mathrm{N}=12)\end{array}$ & $\begin{array}{c}\mathrm{t}- \\
\text { test }\end{array}$ & $\begin{array}{c}\text { p- } \\
\text { valu } \\
\text { e }\end{array}$ \\
\hline \multicolumn{5}{|l|}{$\begin{array}{l}\text { Preoperat } \\
\text { ive }\end{array}$} \\
\hline Mean \pm SD & $\begin{array}{c}2.13 \pm 0.2 \\
3\end{array}$ & $2.27 \pm 0.25$ & \multirow{2}{*}{$\begin{array}{l}0.8 \\
79\end{array}$} & \multirow{2}{*}{$\begin{array}{c}0.52 \\
3\end{array}$} \\
\hline Range & $1.74-2.36$ & $1.84-2.53$ & & \\
\hline \multicolumn{5}{|l|}{$\begin{array}{l}\text { After } 2 \\
\text { wks } \\
\end{array}$} \\
\hline Mean \pm SD & $\begin{array}{c}1.63 \pm 0.1 \\
8 \\
\end{array}$ & $1.61 \pm 0.18$ & \multirow{2}{*}{$\begin{array}{l}1.6 \\
21\end{array}$} & \multirow{2}{*}{$\begin{array}{c}0.96 \\
5\end{array}$} \\
\hline Range & $1.29-1.85$ & $1.24-1.86$ & & \\
\hline \multicolumn{5}{|l|}{$\begin{array}{l}\text { After 1 } \\
\text { month }\end{array}$} \\
\hline Mean \pm SD & $\begin{array}{c}1.31 \pm 0.1 \\
4\end{array}$ & $1.20 \pm 0.13$ & \multirow{2}{*}{$\begin{array}{l}0.8 \\
77\end{array}$} & \multirow{2}{*}{$\begin{array}{c}0.52 \\
2\end{array}$} \\
\hline Range & $1.01-1.50$ & $0.89-1.41$ & & \\
\hline \multicolumn{5}{|l|}{$\begin{array}{l}\text { After } 3 \\
\text { months }\end{array}$} \\
\hline Mean \pm SD & $\begin{array}{c}1.26 \pm 0.1 \\
4 \\
\end{array}$ & $1.06 \pm 0.11$ & \multirow{2}{*}{$\begin{array}{l}0.8 \\
60\end{array}$} & \multirow{2}{*}{$\begin{array}{c}0.51 \\
2\end{array}$} \\
\hline Range & $0.94-1.49$ & $0.72-1.20$ & & \\
\hline
\end{tabular}

t-Independent Sample t-test; p-value >0.05 NS
Table (6) shows no statistically significant difference between radiofrequency powered segmental ablation and endovenous laser ablation according to pain score.

Table (7): Comparison between groups according to radiofrequency powered segmental ablation and endovenous laser ablation according to postoperative pain medication.

\begin{tabular}{|l|c|c|c|c|}
\hline $\begin{array}{c}\text { Postoperati } \\
\text { ve pain } \\
\text { medication }\end{array}$ & $\begin{array}{c}\text { RPSA } \\
(\mathbf{N = 1 2})\end{array}$ & $\begin{array}{c}\text { EVLA } \\
(\mathbf{N = 1 2})\end{array}$ & $\mathbf{x 2}$ & $\begin{array}{c}\text { p- } \\
\text { valu } \\
\mathbf{e}\end{array}$ \\
\hline $\begin{array}{l}\text { No } \\
\text { medication }\end{array}$ & $9(75 \%)$ & $\begin{array}{c}8 \\
(66.7 \%)\end{array}$ & $\begin{array}{c}0.2 \\
97\end{array}$ & $\begin{array}{c}0.77 \\
2\end{array}$ \\
\hline $1-5$ units & $\begin{array}{c}2 \\
(16.7 \%)\end{array}$ & $3(25 \%)$ & $\begin{array}{c}0.2 \\
97\end{array}$ & $\begin{array}{c}0.77 \\
2\end{array}$ \\
\hline $6-10$ units & $1(8.3 \%)$ & $1(8.3 \%)$ & $\begin{array}{c}0.0 \\
00\end{array}$ & $\begin{array}{c}1.00 \\
0\end{array}$ \\
\hline$>10$ units & $0(0 \%)$ & $0(0 \%)$ & $\begin{array}{c}0.0 \\
00\end{array}$ & $\begin{array}{c}1.00 \\
0\end{array}$ \\
\hline
\end{tabular}

$\mathrm{x}^{2}$ : Chi-square test; $\mathrm{p}$-value $>0.05 \mathrm{NS}$

Table (7) shows no statistically significant difference between radiofrequency powered segmental ablation and endovenous laser ablation according to postoperative pain medication.

Table (8): Comparison between groups according to radiofrequency powered segmental ablation and endovenous laser ablation according to freedom from recanalization of great saphenous vein (GSV) observed by duplex ultrasound (DUS).

\begin{tabular}{|l|c|c|c|c|}
\hline & $\begin{array}{c}\text { RPSA } \\
(\mathbf{N = 1 2})\end{array}$ & $\begin{array}{c}\text { EVLA } \\
(\mathbf{N = 1 2})\end{array}$ & $\mathbf{x 2}$ & $\begin{array}{c}\text { p- } \\
\text { value }\end{array}$ \\
\hline At 0 & $12(100 \%)$ & $12(100 \%)$ & $\begin{array}{c}0.0 \\
00\end{array}$ & 1.000 \\
\hline $\begin{array}{l}\text { At 1 } \\
\text { month }\end{array}$ & $\begin{array}{c}11 \\
(91.7 \%)\end{array}$ & $12(100 \%)$ & $\begin{array}{c}0.2 \\
97\end{array}$ & 0.772 \\
\hline $\begin{array}{l}\text { At 3 } \\
\text { months }\end{array}$ & $\begin{array}{c}10 \\
(83.3 \%)\end{array}$ & $\begin{array}{c}11 \\
(91.7 \%)\end{array}$ & $\begin{array}{c}0.2 \\
97\end{array}$ & 0.772 \\
\hline
\end{tabular}

$\mathrm{x}^{2}$ : Chi-square test; $\mathrm{p}$-value $>0.05 \mathrm{NS}$

Table (8) shows no statistically significant difference between radiofrequency powered segmental ablation and endovenous laser ablation according to freedom from recanalization 
of great saphenous vein (GSV) observed by duplex ultrasound (DUS).

Table (9): Comparison between groups according to radiofrequency powered segmental ablation and endovenous laser ablation according to post-operative bruises and ecchymosis and skin burn.

\begin{tabular}{|l|c|c|c|c|}
\hline $\begin{array}{c}\text { Post-operative } \\
\text { bruises and } \\
\text { ecchymosisand } \\
\text { skin burn }\end{array}$ & $\begin{array}{c}\text { RPSA } \\
(\mathbf{N}=12)\end{array}$ & $\begin{array}{c}\text { EVLA } \\
(\mathbf{N}=12)\end{array}$ & x2 & $\begin{array}{c}\text { p- } \\
\text { val } \\
\text { ue }\end{array}$ \\
\hline At 0 & $\begin{array}{c}2 \\
(16.7 \%)\end{array}$ & $\begin{array}{c}1 \\
(8.3 \%)\end{array}$ & $\begin{array}{c}0.3 \\
00\end{array}$ & $\begin{array}{c}0.58 \\
9\end{array}$ \\
\hline At 1 month & $0(0 \%)$ & $0(0 \%)$ & -- & -- \\
\hline At 3 months & $0(0 \%)$ & $0(0 \%)$ & -- & -- \\
\hline
\end{tabular}

$\mathrm{x}^{2}$ : Chi-square test; $\mathrm{p}$-value $>0.05 \mathrm{NS}$

Table (9) shows no statistically significant difference between radiofrequency powered segmental ablation and endovenous laser ablation according toPost-operative bruises and ecchymosis and skin burn.

\section{DISCUSSION}

The ideal treatment for lower extremities primary varicose veins should be relatively noninvasive, repeatable if necessary, relatively safe and free from significant complications, effective in eliminating points of leakage, cost effective, cosmetically acceptable and obviate the necessity for extended periods of unemployment or absence from usual daily activities ${ }^{(8)}$.

Surgical treatment in the form of disconnection of the sapheno-femoral junction (SFJ) along with stripping of the great saphenous vein (GSV) and multiple phlebotomies is considered the standard treatment of varicose veins ${ }^{(9)}$. Excellent results are achieved as long as every source of reflux is eliminated.

There are newer techniques available to destroy the GSV in the thigh, without physically removing the vein by stripping. The alternative techniques in common use are radiofrequency ablation (RFA) and endovenous laser ablation (EVLA). There is now reasonable evidence that radiofrequency ablation is a slightly superior procedure especially for perioperative bruising and pain when compared with endovenous ablation ${ }^{(10)}$.

Twenty four patients were included in our study, 12 patients underwent RFSA, of which there were 3 male $(25 \%)$ and 9 female (75\%) and 12 patients underwent EVLA, of which there were 4 male $(33.3 \%)$ and 8 female $(66.7 \%)$

There was no significant
difference between radiofrequency
powered segmental ablation and
endovenous laser ablation according to
demographic data.

All patients were symptomatic for their venous problems, with or without skin changes. The majority were in the $\mathrm{C} 3$ group of the CEAP classification (8 (66.7\%) patients in RPSA surgery group and $8(66.7 \%)$ in RFA group, followed by C2 (3 (25\%) patients in RPSA group and 2(16.7) patients in EVLA group), and C4 (1(8.3\%) patient in RFA group and 2 (16.7\%) patients in EVLA group).

Our study shows no statistically significant difference between radiofrequency powered segmental ablation (40.95 \pm 6.14$)$ and endovenous laser ablation (38.85 \pm 5.83$)$ according to length of treated vein $(\mathrm{cm})$.

Our study shows no statistically significant difference between radiofrequency powered segmental ablation $12(100 \%)$ and endovenous laser ablation 12 (100\%) according to GSV with incompetent terminal valve.

Our study shows highly statistically significant decrease severity score through the periods in group radiofrequency powered segmental ablation and group endovenous laser ablation.

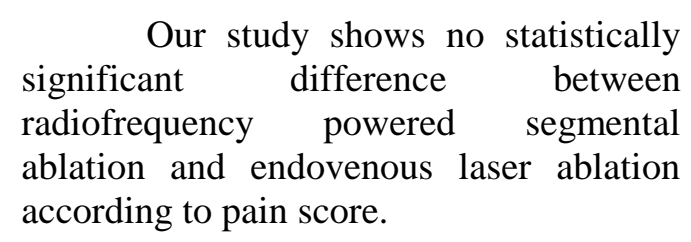

Our study shows no statistically significant difference between radiofrequency powered segmental according to pain score. 
Our study shows highly statistically significant decrease pain score through the periods in group radiofrequency powered segmental ablation and group endovenous laser ablation.

Our study shows no statistically significant difference between radiofrequency powered segmental ablation (no medication) 9 (75\%) and endovenous laser ablation (no medication) $8 \quad(66.7 \%)$ according to postoperative pain medication.

$\begin{array}{ccr}\text { Our study shows no statistically } \\ \text { significant } & \text { difference } & \text { between }\end{array}$ radiofrequency powered segmental ablation At 012 (100\%), At 1 month 11 (91.7\%) and At 3 months 10 (83.3\%) and endovenous laser ablation At 012 (100\%), At 1 month 12 (100\%) and At 3 months 11 (91.7\%) according to freedom from recanalization of great saphenous vein (GSV) observed by duplex ultrasound (DUS).

$\begin{gathered}\text { Our study shows no statistically } \\ \text { between } \\ \text { dignifficant } \\ \text { radiofrequency } \\ \text { powered }\end{gathered}$
ablation At $02(16.7 \%)$, At 1 month 0
$(0 \%)$ and At 3 months $0(0 \%)$ and
endovenous laser ablation At $01(8.3 \%)$,
At 1 month $0(0 \%)$ and At 3 months 0
$(0 \%)$ according to Post-operative bruises
and ecchymosis and skin burn.

\section{CONCLUSION}

Endovenous GSV ablation with Closure Fast and 1470-nm EVLA with RTF had similarly short term outcomes and were equally effective clinically with durable gains in disease-specific QOL. The treatments were equally associated with minimal postprocedural pain scores and short recovery times.

\section{REFERENCES}

1. Jacobs BN, Andraska EA, Obi AT, Wakefield TW (2017): Pathophysiology of Varicose Veins. J Vasc Surg Venous Lymphat Disord., 5 (3): 460-467.
2. El-Sheikha J, Nandhra S, Carradice D, Wallace T, Samuel N, Smith GE, Chetter IC (2014): Clinical outcomes and quality of life 5 years after a randomized trial of concomitant or sequential phlebectomy following endovenous laser ablation for varicose veins. British Journal of Surgery, 101(9): 1093-1097.

3. Tisi PV (2011): Varicose veins. BMJ Clinical Evidence, 2:3.

4. Beale RJ and Gough MJ (2005): Treatment options for primary varicose veins--a review. European journal of Vascular and Endovascular Surgery: the Official Journal of the European Society for Vascular Surgery, 30(1):83-95.

5. Willenberg T, Smith PC, Shepherd $A$ and Davies AH (2013): Visual disturbance following sclerotherapy for varicose veins, reticular veins and telangiectasias:a systematic literature review. Phlebology / Venous Forum of the Royal Society of Medicine, 28(3): 123-31.

6. Leopardi D, Hoggan BL, Fitridge RA, Woodruff PW and Maddern GJ (2009): Systematic review of treatments for varicose veins. Annals of Vascular Surgery, 23(2):264-76.

7. Ladwig A, Riebe H, Pannier F, Schuller-Petrovic $S$ and Junger $M$ (2012): [Endovenous thermal ablation of saphenous varicosis]. Der Hautarzt; Zeitschrift fur Dermatologie, Venerologie, und verwandte Gebiete, 63(8):622-6.

8. Kakkos SK1, Bountouroglou DG, Azzam M, Kalodiki E, Daskalopoulos M, Geroulakos G (2006): Effectiveness and safety of ultrasound-guided foam sclerotherapy for recurrent varicose veins: immediate results. J Endovasc Ther., 13(3):357-64. 
Role of Endovenous Laser Ablation....

9. Neglen $P$ (2001): Long saphenous vein stripping is favored in treating varicose veins. Dermatol Surg., 27:901-902.
10. Nordon IM, Hinchliffe RJ, Brar $\mathbf{R}$ (2011): A prospective double-blind randomized controlled trial of radiofrequency ablation versus laser treatment of the great saphenous vein in patients with varicose veins. Ann Surg., 254:876-881. 